Canadian Journal of Civil Engineering

Canadian

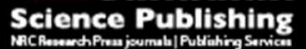

Revue canadienne de génie civil

\title{
Copula Parameter Estimation Using Bayesian Inference for Pipe Data Analysis
}

\begin{tabular}{|r|l|}
\hline Journal: & Canadian Journal of Civil Engineering \\
\hline Manuscript ID & cjce-2017-0084.R1 \\
\hline Manuscript Type: & Article \\
\hline Date Submitted by the Author: & 23-Jun-2017 \\
\hline $\begin{array}{r}\text { Complete List of Authors: } \\
\text { Is the invited manuscript for } \\
\text { consideration in a Special } \\
\text { Issue? : }\end{array}$ & N/A \\
\hline Keyword: & pipe data; copula; Bayesian \\
\hline & \\
\hline
\end{tabular}




\section{Copula Parameter Estimation Using Bayesian Inference for Pipe Data Analysis}

2 Farzana Atique ${ }^{1}$, P.E., and Nii Attoh-Okine², P.E.

3 Abstract: Water main systems are aging and becoming a growing concern for maintenance. The

4 structural deterioration of water mains is affected by different factors, such as pipe age, pipe

5 material, soil condition, and pipe size, among others. Various methods of modeling have been

6 used to predict the failure of water mains. Since pipe networks are underground and obtaining

7 data on pipe conditions is very costly, statistical modeling has been widely used for pipe

8 condition assessment. An emerging statistical method known as copula modeling is used here

9 for pipe data analysis. The copula method is very useful in cases where marginals belong to

10 different families of distributions. It is also useful for generating a large number of data points

11 when it is difficult to obtain a data set, as is the case for pipe condition assessment, and where

12 data sets have random variables belonging to non-Gaussian family distributions. Different

13 copula families are applied here to model the dependency between the pipe age and repair age of

14 pipes. The paper uses a Bayesian framework to estimate the parameter values in the copula model. This approach offers an additional option for estimating copula parameters for pipe data.

Keywords: pipe data, copula modelling, Bayesian statistics

Introduction

The underground water infrastructure system in the United States was installed mainly in the periods of the 1800s, 1900-1945, and post-1945. These pipelines are aging and will start to fail in the coming decades due to age, corrosion, and improper installation. In 2013, The American

21 Society of Civil Engineers (ASCE) issued an infrastructure report card. Drinking water and wastewater infrastructure received D grades (ASCE 2013). 
25 in water supply disruptions, impediments to emergency responses, and damage to other types of 26 infrastructure. Contamination of water due to broken pipes is also a health concern. Broken water

27 pipes can damage roadways and structures. They can also cause disruption in fire control

28 measures. Unscheduled pipe repair work can cause transportation and commerce disruption 29 (ASCE 2013).

Infrastructure asset management is an approach which can help maintain utility at a desired level of service at the lowest life-cycle cost. Asset management practices applied to underground infrastructure can help utility companies understand the timing and cost associated with rehabilitating, repairing, and replacing pipelines. Knowledge gained from these efforts also helps to develop pipe material selection criteria as part of the replacement strategy (Folkman 2012). Knowing the condition of the pipelines and making accurate predictions about the repair and maintenance work is vital for conducting proper asset management for pipe infrastructure. and their eventual failure are caused by static (pipe material, size, age, soil type, loads on pipe, installation quality, groundwater table, etc.) and dynamic (climate, pressure zone change, etc.)

40 factors. The physical mechanism that leads to pipe breakage is a complex process. Pipes are

41 buried underground, and as such there is not enough data to understand the deterioration

42 mechanism. Information on the current condition of the water main along with an understanding

43 of the failure mode can help utility companies better manage their assets in a cost-effective

44 manner. Data required for physical models are costly to obtain. However, high costs can be

45 justified for major transmission mains. For small diameter pipe networks, other methods such as 46 statistical analysis models are more cost effective.

47 One of the emerging methods of statistical modeling, being used in financial sectors and 
48 in hydrological engineering is Copula. Copulas are useful for generating joint distributions by

49 combining given marginal distributions according to a specified form of a copula function.

50 Copulas are appealing because they capture dependencies more broadly than the standard

51 multivariate normal framework. This paper uses copulas to model the bivariate distribution by

52 drawing samples from the joint distributions of dependent variables. The paper then uses the

53 Bayesian statistical method using Markov Chain Monte Carlo (MCMC) sampling to obtain the

54 copula parameter.

\section{Background}

56 There are three modeling procedures used to predict pipe failures (Opila and Attoh-Okine 2011):

57 descriptive analysis models, physical/mechanical models, and statistical models. Descriptive

58 analysis models summarize pipe failure data, which can be used to determine trends in pipe

59 failures. These types of models can provide information on the breakage rates for groups of pipes

60 but not information about individual pipes.

Physical models use the physical mechanism that leads to pipe failure to predict future

62 pipe failures. The physical mechanisms that affect pipe failure are influenced by the pipe

63 structural properties, internal and external loads and material deterioration. The data required for

64 physical models are hard to obtain. Some physical model data include continuous freezing index,

65 soil backfill properties such as porosity, segregation potential, unfrozen water content and

66 thermal gradient at the freezing, elastic soil reaction modulus, the pipe-soil reaction modulus etc

67 (Kleiner and Rajani 2001b). The high cost of obtaining data makes these models appropriate for

68 large lines but very costly for smaller pipe networks. The third type, the statistical models, has

69 been widely used for pipe failure modeling. Kleiner and Rajani (2001a), have summarized and

70 provided list of the statistical models that have been 
71 proposed for water mains condition assessment. The statistical methods for predicting water

72 main breaks use historical data on past failures to predict a future pipe breakage pattern. The

73 statistical models are classified into two groups: deterministic and probabilistic models. The

74 deterministic models predict breakage rates using two or three parameters (Opila and Atoh-

75 Okine 2011). Many other factors affect the breakage of pipes and to incorporate other factors,

76 pipes are classified into groups that are uniform and homogeneous with respect to factors not

77 included in the models. These models use the grouping criteria as covariates in the analysis while

78 retaining a simple mathematical framework. Probabilistic single-variate models use probabilistic

79 processes on grouped data to derive probabilities of pipe life expectancy, probabilities of

80 breakage, and the probability of break clustering phenomenon. The probabilistic multivariate

81 models can consider most of the covariates in the analysis. They do not require the pre-

82 partitioning of the data. Table 1 shows some of the statistical failure models.

83 Table 1. Sample statistical failure models (adapted from Opila and Attoh-Okine 2011)

\begin{tabular}{lll}
\hline Deterministic models & $\begin{array}{l}\text { Probabilistic single variate } \\
\text { models }\end{array}$ & $\begin{array}{l}\text { Probabilistic multivariate } \\
\text { models }\end{array}$ \\
\hline Counting process & Cohort-survival models & Accelerated lifetime models \\
Time-exponential models & Semi-Markov process & $\begin{array}{l}\text { Time-dependent Poisson } \\
\text { models }\end{array}$ \\
Time linear models & Break clustering & Neural networks \\
Generalized linear model & Bayesian diagnostic & Proportional hazard models \\
& & (PHM) \\
& & -Bathtub effect PHM \\
& & -Poisson PHM \\
& & -Weibull PHM \\
\hline
\end{tabular}

84

Copula modeling is an emerging method of statistical modeling. Even though the use of copulas has become dominant in actuarial science, the financial sector, and biology, there has been limited use of copula modeling in the civil engineering research field. There have been a few research papers on copula modeling in hydrology (Genest and Favre 2007), infrastructure 
89 dependence modeling (Attoh-Okine 2013), water pipe dependence modeling (Atique and Okine 90 2016), and modelling of vehicle axle-weight (Srinivas et al. 2006).

In this paper, copula dependence modeling is introduced to pipe condition data. The

92 study of multivariate distributions has been dominated by the normal distribution (Frees and

93 Valdes 1998). The choice of the multivariate normal distribution is appealing as the marginal

94 distributions are also normal and the association between two random variables can be fully

95 described by knowing a) their marginal distribution and b) the correlation coefficient. However,

96 in engineering applications, non-normality can occur in the following ways: a) the marginal

97 distribution of some of the variables may not be normal or b) in some cases, even though all the marginal distributions are normal, jointly these variables may not be multivariate normal (Yan

99 2006). Copulas are useful for generating joint distributions by combining given marginal distributions according to a specified form of a copula function. Copulas are appealing because

101 they capture dependence more broadly than the standard multivariate normal framework.

Linear correlation is used as a measure of dependence between variables following a multivariate normal or elliptical distribution. The dependence measure most frequently used is

104 Pearson's correlation coefficient, which is related with linear dependence and the normal 105 distribution (Accioly and Chiyoshi 2004). However, individual infrastructure conditions may 106 have fat tails, skewness, and non-normal characteristics (Attoh-Okine 2013). This makes 107 inference of the dependency based on correlation inaccurate for non-Gaussian data.

108 The copula approach uses Kendall's rank correlation and Spearman's rank correlation, which 109 measure dependence across the entire distribution, as opposed to Pearson's mean correlation, and 110 are able to model tail dependencies in distributions. The advantages of copula functions have 111 been summarized as follows (Srinivas et al. 2006): 
112 1) This process is useful when multivariate simulation is to be carried out from dependent

113 random variables from different classes of marginal distributions

114 2) The copula approach provides a way to separate marginals from the dependence

115 structure, hence reducing the study of a multivariate distribution to a study of a

116 multivariate dependence structure.

117 3) The limitation of using the linear correlation coefficient is avoided.

118 4) The invariance property of the copula dependence structure under transformations and its 119 independence from the marginal distribution can provide efficient simulations.

121 Berg (2008) has listed the following features of copula modeling:

122 1) The copula contains all the information about the dependence between random variables.

123 2) Copulas provide an alternative and more useful representation of multivariate distribution 124 functions compared to traditional approaches such as multivariate normality.

125 3) Most traditional representations of dependence are based on the linear correlation

4) Copulas enable marginal distributions and the dependence structure to be modeled separately.

5) Copulas provide greater modeling flexibility; given a copula, we can obtain many multivariate distributions by selecting different margins.

132 6) Any multivariate distribution can serve as a copula.

133 7) A copula is invariant under strictly increasing transformations. 
134 135

8) Most traditional measures of dependence are measures of pairwise dependence. Copulas measure the dependence between all $d$ random variables. Kelly (2007) used copulas to model dependence in simulation risk assessment. The paper used the copula as a tool for risk assessment, stating that common-cause failure models have limitations and substantial subjectivity is associated with parameter estimation for those models. The paper applied the Marshall-Olkin copula and the Frank copula for analysis and stated that marginal distributions in a joint distribution will not be Gaussian; typically, they will be exponential, Weibull, gamma, or lognormal, which complicates the process of drawing Monte Carlo samples from the joint distribution. Romeo and Tanaka (2006) proposed a Bayesian modeling for bivariate survival data and used it for a diabetic retinopathy study. Their method of analysis included slice sampling and the Metropolis Hastings algorithm to sample the distribution. Silva and Lopes (2008) used a Bayesian approach to estimate the parameters of copula models using a two-step method by fitting empirical distribution functions to marginals and maximum likelihood to the copula model.

\section{Copulas}

Copulas were introduced in 1959 by Sklar. An $n$-dimensional copula is a multivariate distribution function defined on the unit cube $[0,1]^{\mathrm{n}}$ with uniformly distributed marginals. The idea of dependence modeling with copula functions is based on Sklar's theorem (Srinivas et al. 2006). It states that if $\mathrm{H}$ is an $n$-dimensional cumulative distribution function with continuous marginal cumulative distributions $\mathrm{F}_{1}, \mathrm{~F}_{2}, \ldots, \mathrm{F}_{\mathrm{n}}$, then there exists an $n$-dimensional copula $\mathrm{C}$ such that for all the real values of $s=\left[\mathrm{s}_{1} \mathrm{~s}_{2} \ldots . . \mathrm{s}_{\mathrm{n}}\right]^{\mathrm{T}}$,

$$
H(s)=C\left(F_{1}\left(s_{1}\right), F_{2}\left(s_{2}\right), \ldots \ldots, F_{n}\left(s_{n}\right)\right)
$$

Where $\mathrm{C}$ is unique for marginal distribution functions which are continuous. 
Sklar's theorem also shows that for an $n$-dimensional joint distribution function, the $n$

157

$$
\begin{gathered}
h(s)=\frac{\partial^{n}\left[C\left(F_{1}\left(s_{1}\right), \ldots, F_{n}\left(s_{n}\right)\right)\right]}{\partial F_{1}\left(s_{1}\right), \ldots \ldots, \partial F_{n}\left(s_{n}\right)} \prod_{i=1}^{n} f_{i}\left(s_{i}\right) \\
h(s)=c\left(F_{1}\left(s_{1}\right), \ldots \ldots \ldots \ldots, F_{n}\left(s_{n}\right)\right) \prod_{i=1}^{n} f_{i}\left(s_{i}\right)
\end{gathered}
$$

162 where $c\left(F_{1}\left(s_{1}\right), \ldots \ldots \ldots, F_{n}\left(s_{n}\right)\right)=$ copula density associated with the copula function $C\left(F_{1}\left(s_{1}\right), \ldots \ldots \ldots, F_{n}\left(s_{n}\right)\right)$ and $f_{i}=$ marginal probability density function corresponding to $F_{i}$

164 (Srinivas et al. 2006).

There are many functions which satisfy the mathematical condition for being a copula 166 normal distributions, and enables modeling of multivariate extremes and other forms of non-

172 normal dependences (Embrechts et al. 2001).

\section{Archimedean Copulas}

174 Archimedean copulas are a very popular copula family and have a wide range of applications

175 because of the following reasons (Bacigal 2006):

176

a. They can be constructed easily. 
b. They can be applied whether the correlation between variables is positive or negative.

178 The Archimedean bivariate copula is expressed as (Nelsen 1999)

$$
C(u, v)=\varphi^{-1}(\varphi(u)+\varphi(v)), \quad 0<u, v<1
$$

having different generators, there are derivations of several important copulas. Some of the

Table 2. Archimedean copulas (adapted from Attoh-Okine 2013)

\begin{tabular}{|c|c|c|c|}
\hline Family of copula & Generator $\emptyset_{t}$ & Parameter $\theta$ & Bivariate copula $C_{\varphi}(u, v)$ \\
\hline Independence & $-\ln t$ & & $u v$ \\
\hline Gumbel & $(-\ln t)^{\theta}$ & $\theta \geq 1$ & $e^{-\left[(-\ln u)^{\theta}+(-\ln v)^{\theta}\right]^{-1 / \theta}}$ \\
\hline Clayton & $t^{-\theta}-1$ & $\theta>0$ & $\left(u^{-\theta}+v^{-\theta}-1\right)^{-1 / \theta}$ \\
\hline Frank & $-\ln \left(\frac{e^{-\theta t}-1}{e^{-\theta}-1}\right)$ & $\theta \in \mathbb{R}$ & $\frac{1}{\theta} \ln (1$ \\
\hline & & & $\left.+\frac{\left(e^{-\theta u}-1\right)\left(e^{-\theta v}-1\right)}{e^{-\theta}-1}\right)$ \\
\hline
\end{tabular}
Gumbel copula is good for positive tail dependence rather than negative tail dependence.

187 Meanwhile, the Frank copula is a symmetric Archimedean copula where tail dependence is weak. The comparisons between elliptical and Archimedean copulas are shown in Table 3. 
Table 3. The comparison between elliptical copulas and Archimedean copulas (adapted from

190 Naimy 2012)

\begin{tabular}{|c|c|c|}
\hline & Elliptical copula & Archimedean copula \\
\hline Definition & $\begin{array}{l}\text { Elliptical copulas are of elliptically } \\
\text { contoured distributions. Widely } \\
\text { known elliptical distributions are: } \\
\text { normal (Gaussian) and student's t } \\
\text { copula. }\end{array}$ & $\begin{array}{l}\text { Archimedean copulas are } \\
\text { easily constructed and have } \\
\text { attractive properties. } \\
\text { Commonly used distributions } \\
\text { are: Clayton, Frank, and } \\
\text { Gumbel. }\end{array}$ \\
\hline Advantages & $\begin{array}{l}\text { The correlation between the } \\
\text { marginals can be easily } \\
\text { determined. }\end{array}$ & They are easily deduced. \\
\hline Disadvantages & $\begin{array}{l}\text { Absence of closed form } \\
\text { expressions and impossible to have } \\
\text { radial symmetry }\end{array}$ & $\begin{array}{l}\text { The definition does not } \\
\text { extend to a multivariate data } \\
\text { set of n variables as there will } \\
\text { be multiple values of tau. }\end{array}$ \\
\hline
\end{tabular}

191

Since Archimedean copulas are easily constructed and deduced, for analysis of copula parameter using Bayesian approach Archimedean copulas are chosen for analysis of pipe data in this research.

\section{Measure of Dependence}

Common measures that are used for analyzing dependence are the 1) Pearson, b) Spearman, and

3) Kendall coefficients. For copula dependence measurements, Spearman's rho and Kendall's $\left(x_{1}, y_{1}\right)$ and $\left(x_{2}, y_{2}\right)$, each with $\mathrm{H}$ distribution (Attoh-Okine 2013):

$$
\tau(x, y)=\operatorname{Pr}\left(\left(x_{1}-x_{2}\right)\left(y_{1}-y_{2}\right)>0\right)-\operatorname{Pr}\left(\left(x_{1}-x_{2}\right)\left(y_{1}-y_{2}\right)<0\right)
$$

where $\left(x_{1}-x_{2}\right)\left(y_{1}-y_{2}\right)>0$ is concordant and $\left(x_{1}-x_{2}\right)\left(y_{1}-y_{2}\right)<0$ is discordant. 
204 Kendall's tau can be expressed as

$$
\tau(x, y)=\frac{\# \text { Concordant pairs-\#Discordant pairs }}{\# \text { pairs }}
$$

205

206

Parameter Estimation

The dependence structure captured by copulas does not have anything to do with the individual behavior of the variables, so any inference about the parameter indexing a family of

209 copulas should rely on the rank of the observations (Genest and Favre 2007). Methods normally used for estimation are estimates based on Kendall's tau, Spearman's rho, and maximum pseudolikelihood. $C_{\theta}$ be continuous with density $c_{\theta}$ and involves maximizing a rank-based, log-likelihood of the form (Genest and Favre 2007)

216

$$
l(\theta)=\sum_{i=1}^{n} \log \left\{c_{\theta}\left(\frac{R_{i}}{n+1}, \frac{s_{i}}{n+1}\right)\right\}
$$

218 where $R_{i}$ stands for the rank of $X_{i}$ among $X_{1}, \ldots \ldots, X_{n}$ and $S_{i}$ stands for the rank of $Y_{i}$ among

$219 Y_{1}, \ldots \ldots, Y_{n}$. Letting $\dot{c_{\theta}}(u, v)=\partial c_{\theta}(u, v) / \partial \theta$, Genest et al. (1995) showed that 220

$$
l \dot{(\theta)}=\frac{\partial}{\partial \theta} l(\theta)=\sum_{i=l}^{n} \frac{c_{\theta}\left(\frac{R_{i}}{n+1}, \frac{s_{i}}{n+1}\right)}{\dot{c}_{\theta}\left(\frac{R_{i}}{n+1}, \frac{s_{i}}{n+1}\right)}=0
$$

222 is unique. 


\section{Basics of Bayesian Inference}

224 Bayes' theorem is based on the conditional probability as denoted by

$$
p(y \mid x)=p(y, x) / p(x)
$$

225 In words, the definition implies that the probability of $y$ given $x$ is the probability that they

226 happen together relative to the probability that $x$ happens at all. Multiplying both sides of Eq. 9

227 by $p(x)$ results in $p(y \mid x) p(x)=p(y, x)$. Similarly, starting with $p(x \mid y)=p(y, x) / p(y)$ results 228 in $p(x \mid y) p(y)=p(y, x)$. With two similar expressions for $p(y, x)$, one finds $p(y \mid x) p(x)=$

$229 p(x \mid y) p(y)$. Dividing both sides by $p(x)$ results in

230

$$
p(y \mid x)=\frac{p(x \mid y) p(y)}{p(x)}
$$

$232 p(x)$ can be expanded as $p(x)=\sum_{y} p(x, y)=\sum_{y} p(x \mid y) p(y)$; substituting this into Eq. 10 233 yields

$$
p(y \mid x)=\frac{p(x \mid y) p(y)}{\sum_{y} p(x \mid y) p(y)}
$$

235 Eq. 10 and Eq. 11 are called Bayes' rule, and this simple relationship lies at the core of Bayesian 236 inference. For a continuous variable, Eq. 11 becomes 


$$
p(y \mid x)=\frac{p(x \mid y) p(y)}{\int d y p(x \mid y) p(y)}
$$

239 believe the model, given the data:

241 For a model with data value D and parameter value $\theta$, Bayes' rule can be expressed as

$$
p(\theta \mid D)=p(D \mid \theta) \quad x \quad p(\theta) / p(D)
$$

244 Where

$$
p(D)=\int d \theta p(D \mid \theta) p(\theta)
$$

246 The prior $p(\theta)$ is the strength of the belief in $\theta$ without the data $\mathrm{D}$. The posterior $p(\theta \mid D)$ is

247 the strength of belief in $\theta$ when the data $\mathrm{D}$ have been taken into account. The likelihood $p(D \mid \theta)$

248 is the probability that the data could be generated with parameter value $\theta$.

249 When there is observed data, Bayes' rule can be used to determine beliefs across competing 250 parameter values in a model and to determine beliefs across competing models. The evidence $251 p(D)$ is the probability of the data according to the model, determined by summing across all 252 possible parameter values weighted by the strength of belief in those parameter values. 
253 For copula parameter estimation, maximum likelihood estimation is often straightforward.

254 However, there are cases where a Bayesian analysis can be preferable. Smith (2011) noted that:

255

256

257

258

259

260

261

262

263

264

265

266

267

268

269

270

271

272

273

- For more complex marginal models and/or copula functions, the likelihood can be hard to maximize directly. One of the solutions mentioned in the paper is to use a two-stage estimator where the marginal model parameters $\theta_{j}$ are estimated first and then $\emptyset$ estimated conditionally on these. Another Bayesian alternative in this circumstance is to construct an inference from the joint posterior $f(\Theta, \emptyset \mid y)$, evaluated in a Monte Carlo manner, with $\Theta$ and $\emptyset$ generated separately in a Gibbs sampling scheme.

- Bayesian hierarchical modeling has been proven successful for the modeling of multivariate data.

- When estimating a copula model, the objective is often to construct inferences on the measures of dependence, quantiles, and/or functionals of random variable vector $\mathrm{Y}$ or parameters $(\Theta, \varnothing)$. The evaluation of the posterior distribution of these quantities is often easy using Markov Chain Monte Carlo (MCMC) methods.

\section{Pipe Data Analysis Using Copula Modeling}

Figure 1 shows a general flow chart for pipe data analysis using copula method. In the beginning pipe inspection data obtained from methods described in Section 2.2 are combined with pipe inventory data such as pipe length, pipe age etc. If GIS data are available, that can also be used for the pipe data analysis. In the data analysis phase, copula modeling can be used to determine the dependence between variables. In pipe deterioration process there is not only a large number of processes that can lead to the degradation of pipes, but also a correspondingly 
274 large number of exploratory variables (Scheidegger et al. 2011). Pipe construction has evolved 275 through many different construction processes and use of different construction materials. In 276 addition, the site conditions, i.e. soil conditions, water table location, water quality etc. can all 277 affect the rate of deterioration. It is important to find, determine and chose exploratory variables 278 for useful prediction models. For cases where the dependence is non linear and skewed, 279 Spearman's rho and Kendall's tau can be better methods for dependence analysis. Dependence analysis can help to determine the relevancy of pipe deterioration variables. Dependence of water pipe data is covered in paper Atique and Okine (2016). Generated large data sets can be used for different objectives. Based on bivariate copula dependence modeling, generated data points are used for regression analysis. Multivariate simulation for copula modeling can also be used to determine the effectiveness and comparison of the existing pipe deterioration models. A set of water pipe data was obtained from a utility company in the desert west region. The data set included pipes ranging from 4" diameter to 66" diameter. The oldest pipe was installed in 1943, and the newest one was installed in 2009. The data notation used for the variables is shown in Table 4. For analysis, 978 observations were used. These pipes had repairs done to them and were in active status. From Material variable $\mathrm{M}$ indicates material type indicated by code. Distribution class varieties include distribution, fire hydrant lateral, service lateral, and transmission. The scatter plots of the variables are shown in Figure 2. For an example, the second column and top row gives the dependency figure for pipe material and pipe age. The $\mathrm{x}$-axis shows the material code from 10 to 60 whereas the pipe age varies from 1 to 40,000. Since the age of the pipe can be a maximum of 24,000 days based on installation day, 40,000 value was an error. The data point is a PVC pipe that had an arbitrary installation date of

$2961 / 1 / 1900$. The scatter plot provides an initial revelation that the variables do not provide joint 297 normal distribution. Marginals of some of the variables are also found to be non-normal in 298 further analysis. This provides a good opportunity to study copula modeling for this set of 299 variables. 
* In case available

\section{Data Analysis}

\section{Pipe inventory data pipe length, diameter, pipe age ntr}
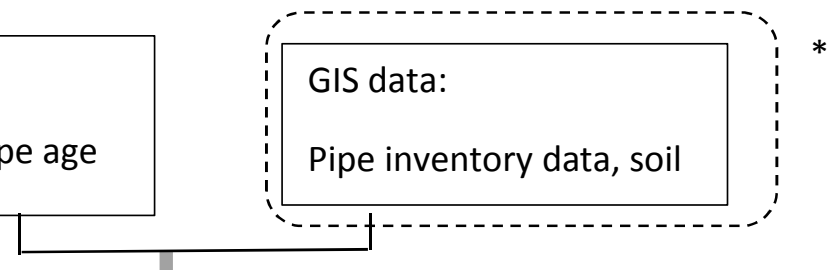

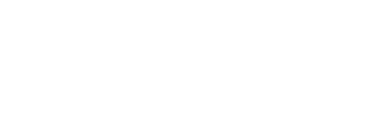
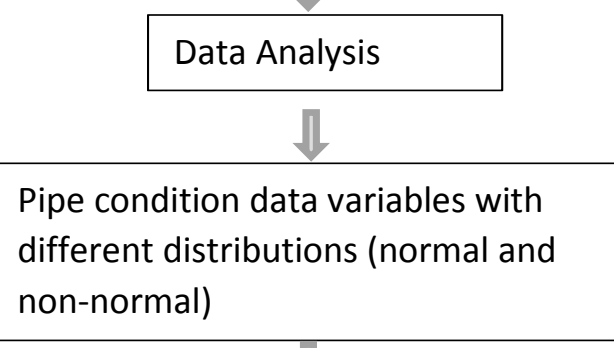

Exploratory

data analysis non-normal)
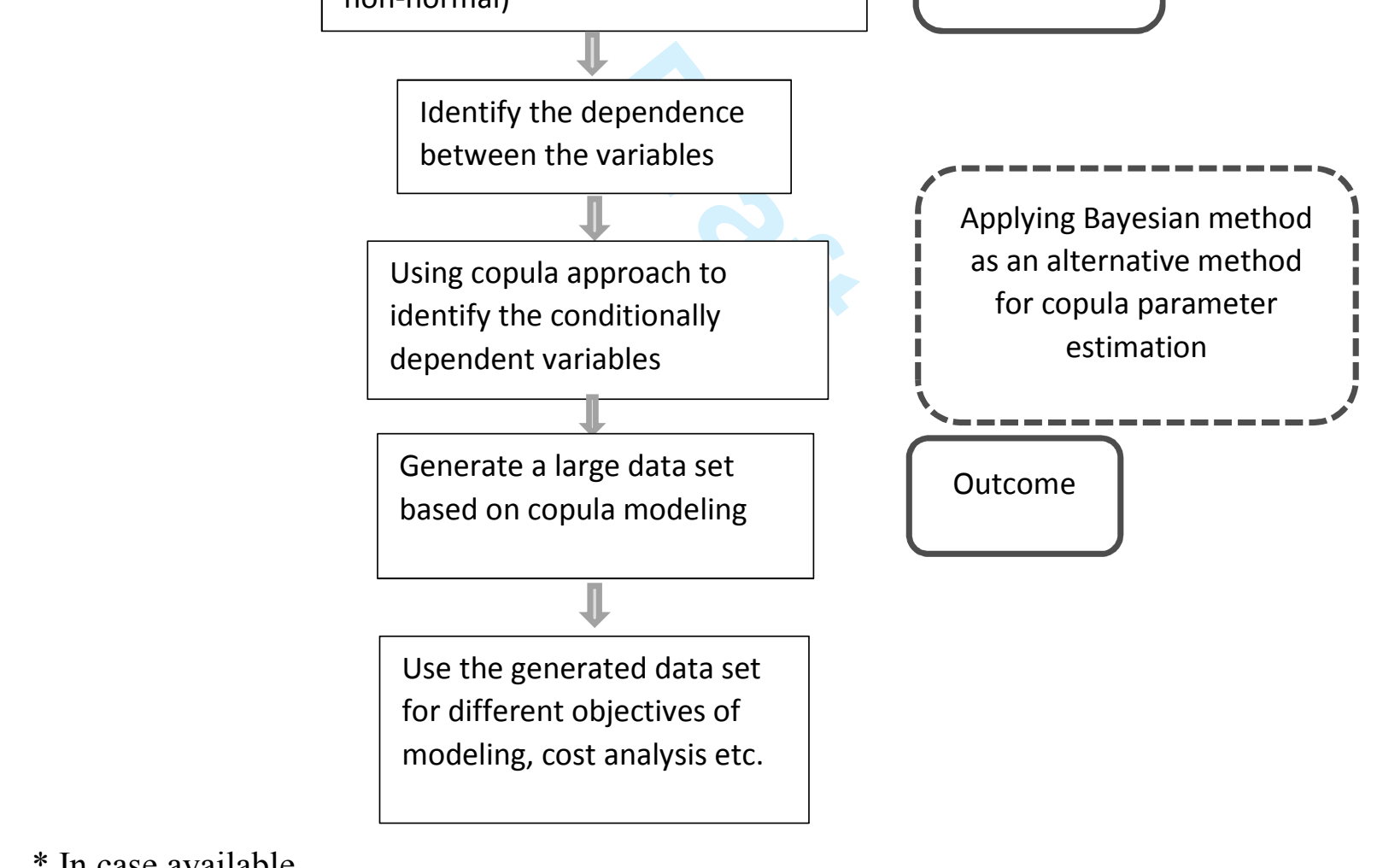
Table 4. Notation used for data analysis

\begin{tabular}{ll}
\hline Variable & Meaning \\
\hline D & Diameter of pipe \\
M & Pipe material (code number indicated different \\
& material) \\
L & Length of pipe \\
PA & Pipe age (in days) \\
RA & Repair age (in days) \\
\hline
\end{tabular}

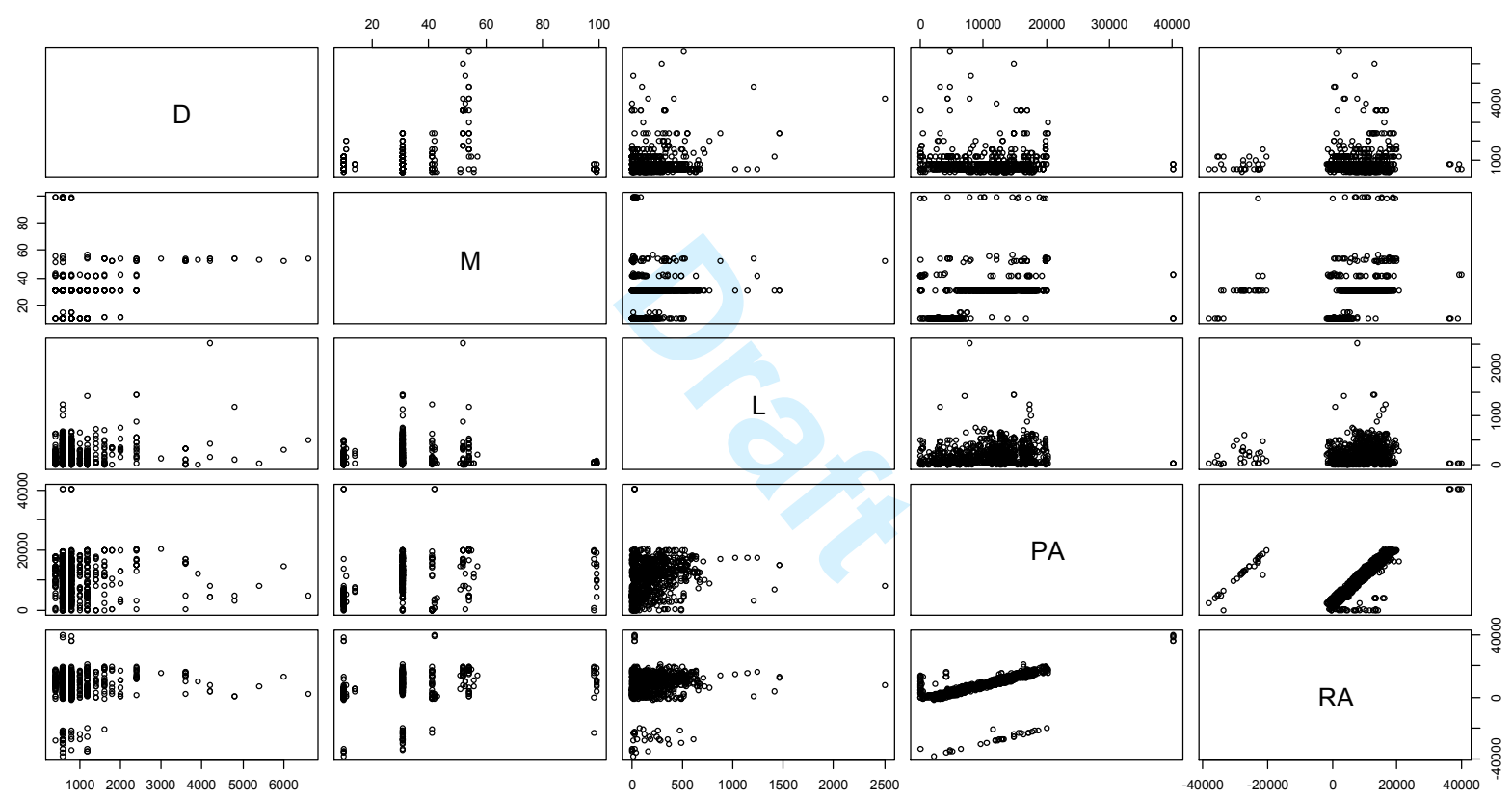

Figure 2. Scatter plots of different variables of pipe data

Pipe age and repair age were the two variables chosen for analysis using the copula method and application of Bayesian inference for parameter analysis. Pipe age is the age

342 recorded at the time the inspection was conducted. Repair age is based on the time the pipe repair

343 took place. The pipe age and repair age were converted to years instead of days for simplicity.

344 The refined data set gave a correlation value for Spearman's rho of 0.936 for pipe age and repair 
345 age. Figure 3 shows the histogram for pipe age and demonstrates that the general extreme value 346 type distribution fits the data well.

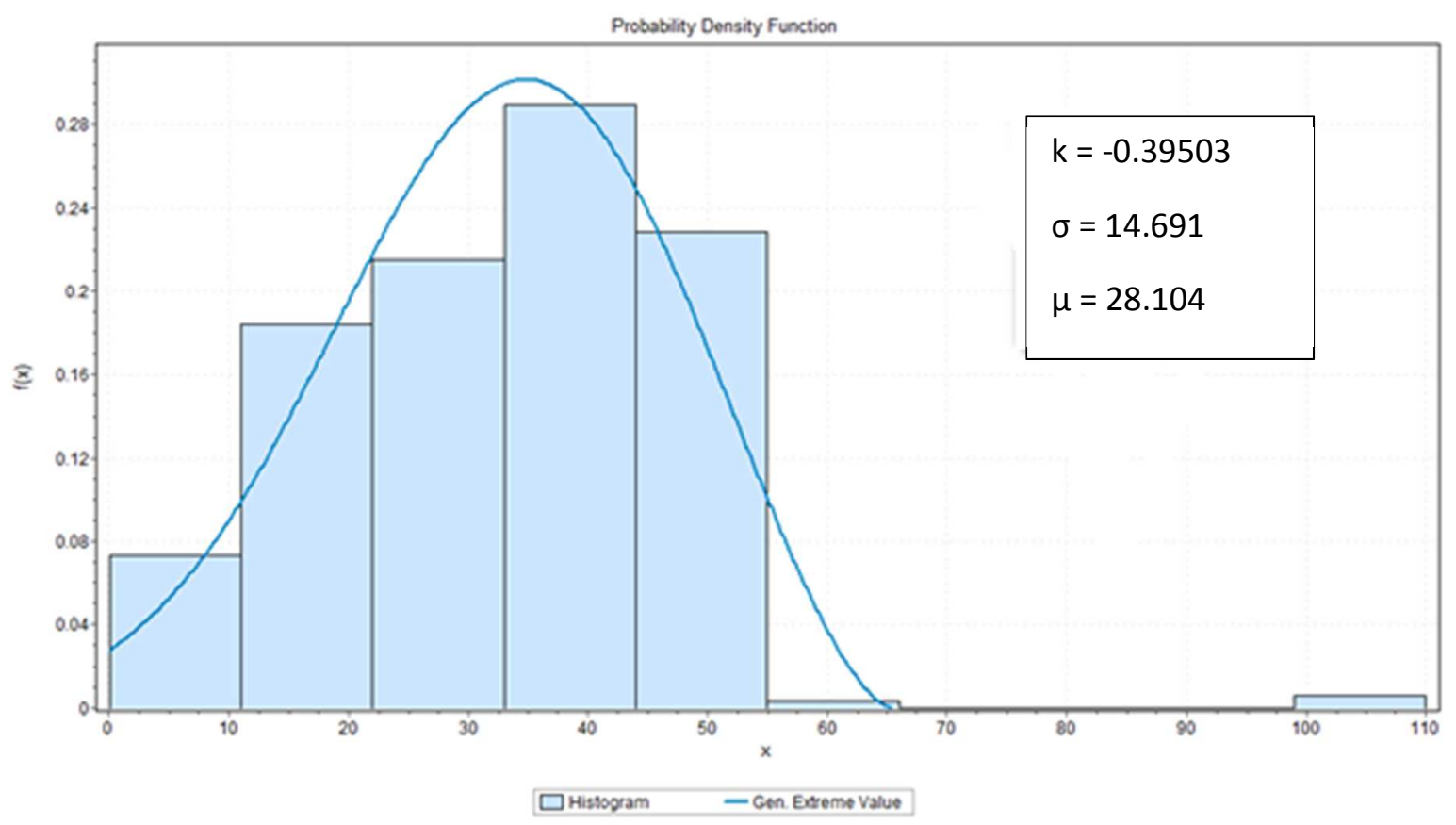

347

348 Figure 3. Pipe age data distribution 


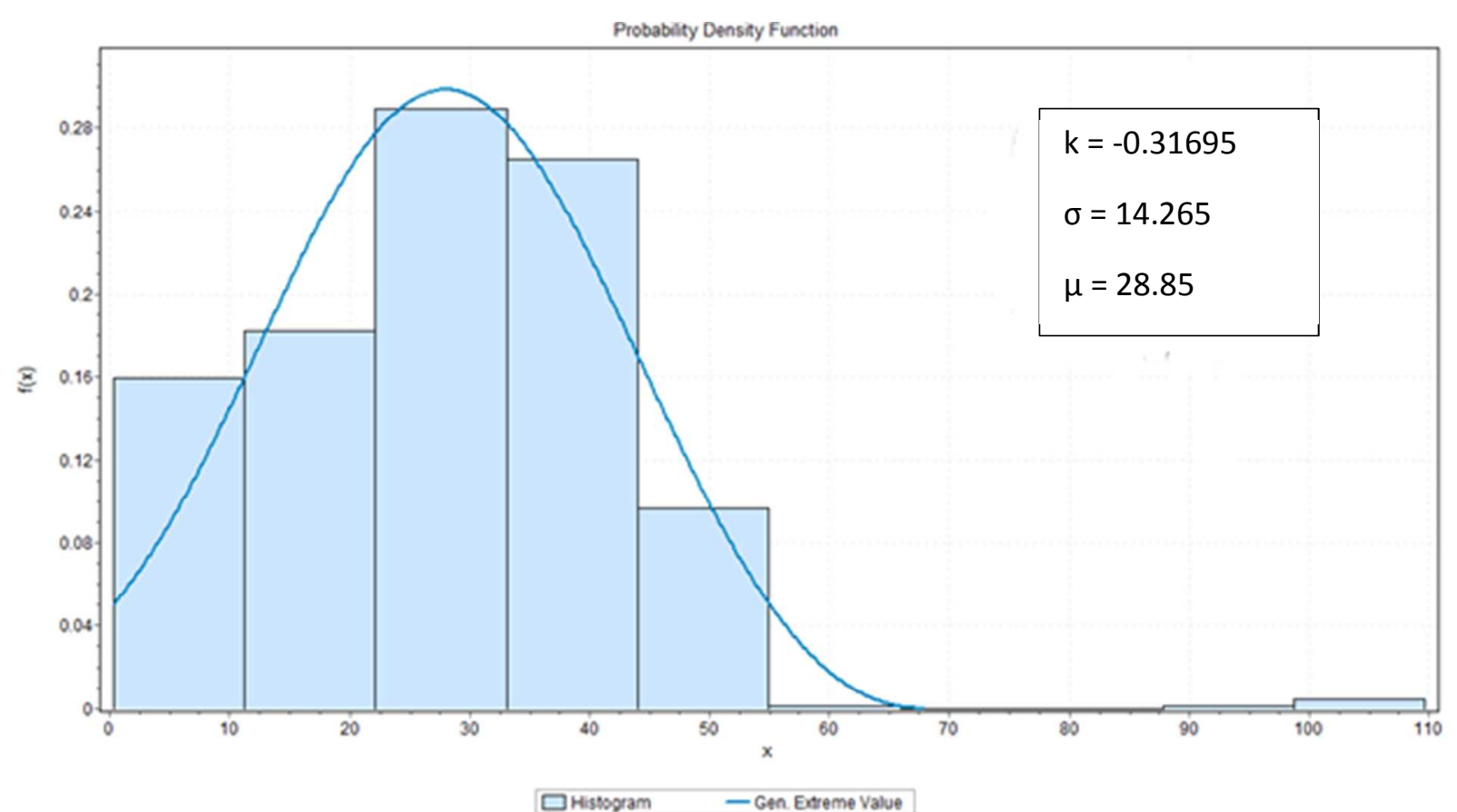

Figure 4. Repair age data distribution

352 was obtained. Using the general extreme value marginal and the Gumbel copula 4.5, 1000 data

353 points were generated. The points were plotted to the original data set as shown in Fig. 5. The 354 figure shows that the generated points match the original points well.

356 sets were used for regression analysis, and the results are shown in Table 5. It is apparent that

357 using the GEV as a marginal gives a better result for regression analysis with an R squared value 358 of 0.88 and a standard error of 4.89 . 


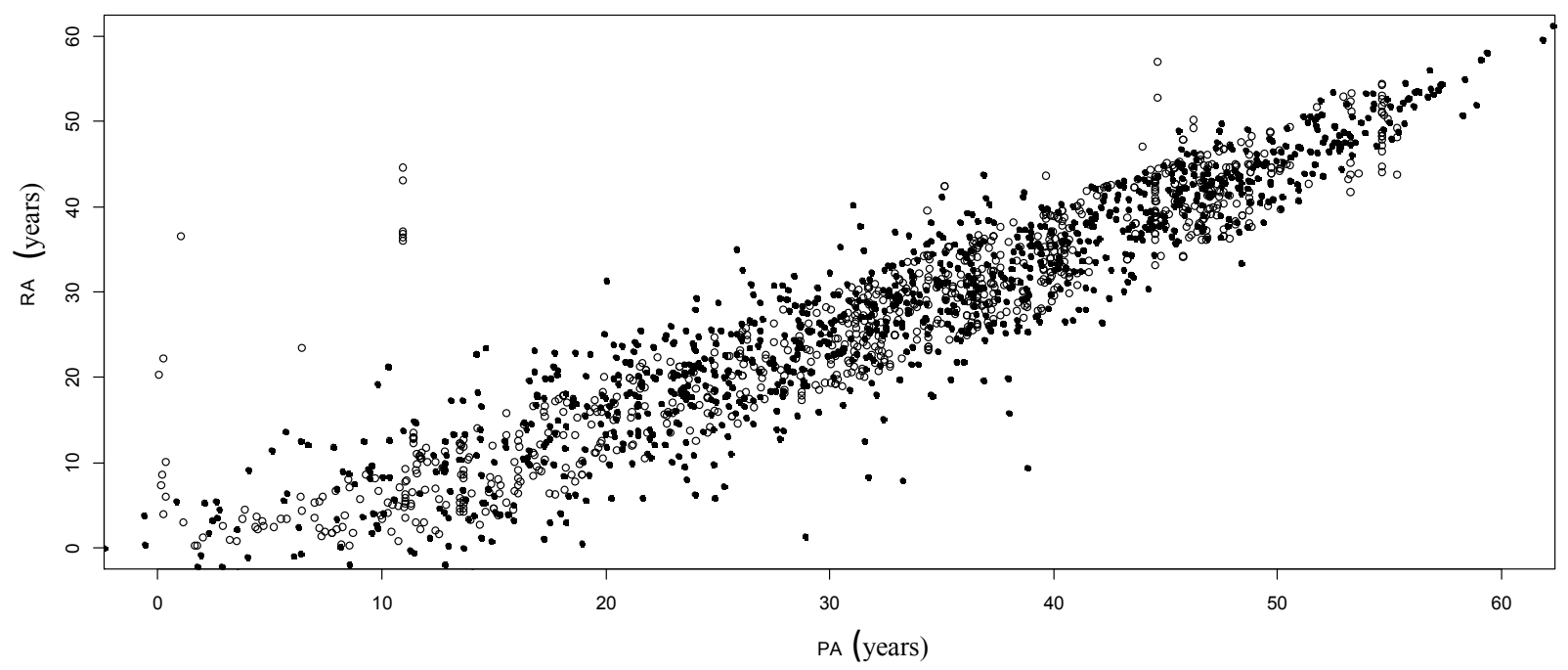

Figure 5. Original data points in hollow circles and copula-generated data in solid circles. PA

362 denotes pipe age, and RA denotes repair age.

Table 5. Comparison of regression analysis using copula modeling and original data

\section{Regression statistics}

Gumbel copula

GEV marginal generated data

Original data points
Gumbel copula normal marginal generated data points

\begin{tabular}{cccc} 
R Squared & 0.88 & 0.88 & 0.87 \\
Standard & & & \\
error & 4.92 & $\mathbf{4 . 8 9}$ & 5.06 \\
Observations & 902 & 1000 & 1000 \\
\hline
\end{tabular}
copula are discussed in the following sections. 
368 From Eq. 13, the likelihood function for Bayesian inference for the copula parameter can also be

369 written as (Kelly 2007)

$$
f\left(t_{1}, t_{2}\right)=f_{1}\left(t_{1}\right) f_{2}\left(t_{2}\right) c\left[F_{1}\left(t_{1}\right), F_{2}\left(t_{2}\right)\right]
$$

370 where $\mathrm{c}$ is the copula density, and for a marginal of normal distribution,

$$
f_{i}\left(t_{i}\right)=\sqrt{\frac{\tau}{2 \pi}} e^{-\frac{\tau_{i}}{2}\left(t_{i}-\mu_{i}\right)^{2}}
$$

371 where $\mu$ denotes the mean of the distribution and $\tau$ is known as precision, which equals $1 / \sigma^{2}$.

373 The "zero tricks" as described in WinBUGS manual is used to encode the likelihood function

374 (Kelly 2007). In zero tricks, the Poisson (phi) observation of zero has likelihood exp(-phi), so if

375 observed data is a set of 0 's and phi[i] is set to $-\log (\mathrm{L}[\mathrm{i}])$, the correct likelihood is obtained.

376 Bivariate Gumbel copula is given by the following formula:

$$
C(u, v ; \theta)=e^{-\left[(-\ln u)^{\theta}+(-\ln v)^{\theta}\right]^{-1 / \theta}}
$$

378 The bivariate copula density

$$
c(u, v)=\frac{\partial^{2} C(u, v)}{\partial u \partial v}
$$

$$
\begin{gathered}
c(u, v ; \theta)=\frac{C(u, v ; \theta)(\log (u) \cdot \log (v))^{\theta-1}}{u v\left((-\log (u))^{\theta}+(-\log (v))^{\theta}\right)^{2-\frac{1}{\theta}}} x\left((-\log (u))^{\theta}\right. \\
\left.\left.+(-\log (v))^{\theta}\right)^{\frac{1}{\theta}}+\theta-1\right)
\end{gathered}
$$


381 where $u=F_{1}\left(t_{1}\right)$ and $v=F_{2}\left(t_{2}\right)$ are in Eq. 19.

For the Gumbel copula, the density function in Eq. 19 was used as a likelihood function in Bayesian analysis. The theta prior of uniform value of 1 to 100 was also used. 100 random data points. The parameter estimated by the maximized pseudolikelihood method yielded a value of 4.95 with a standard error of 0.69 . For the same data set of repair age and pipe 387 age, using a normal marginal resulted in parameter estimation of 4.81 with a standard error of $388 \quad 0.67$.

389 The generated data using a normal marginal was used to obtain the parameter by Bayesian 390 inference. The mean value of the parameter was 4.106 . The range for the 2.5 th percentile was 3913.40 and for the 97.5 th percentile was 4.912, as shown in Table 6. Comparing this theta value 392 with those obtained from the maximized pseudolikelihood method shows that the value obtained 393 from Bayesian inference is of a narrower range than the $95 \%$ confidence interval for the theta 394 value obtained from the maximized pseudolikelihood method as shown in Fig. 6.

395 Table 6. Results for Gumbel copula parameter estimation

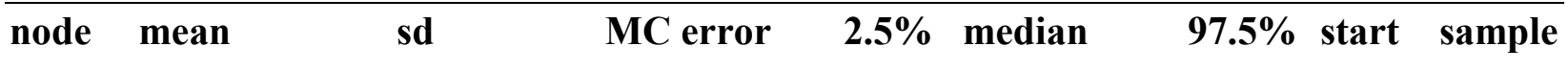

397

mu1 31.56

0.8399

0.04827

$29.81 \quad 31.58$

$33.154001 \quad 6000$

398

mu2 26.77

0.7877

0.04559

$25.2 \quad 26.75$

$28.29 \quad 4001 \quad 6000$

399

taul $\quad 0.004936$

4.293E-4

$\begin{array}{lll}1.128 E-5 & 0.004128 & 0.004922\end{array}$

$0.0058314001 \quad 6000$

400

tau2 0.005282

4.651E-4

$\begin{array}{lll}1.26 \mathrm{E}-5 & 0.004428 & 0.00526\end{array}$

$0.0062524001 \quad 6000$ 
401

theta 4.106

0.3857

0.007917

$3.402 \quad 4.085$

$4.912 \quad 4001 \quad 6000$

402

7

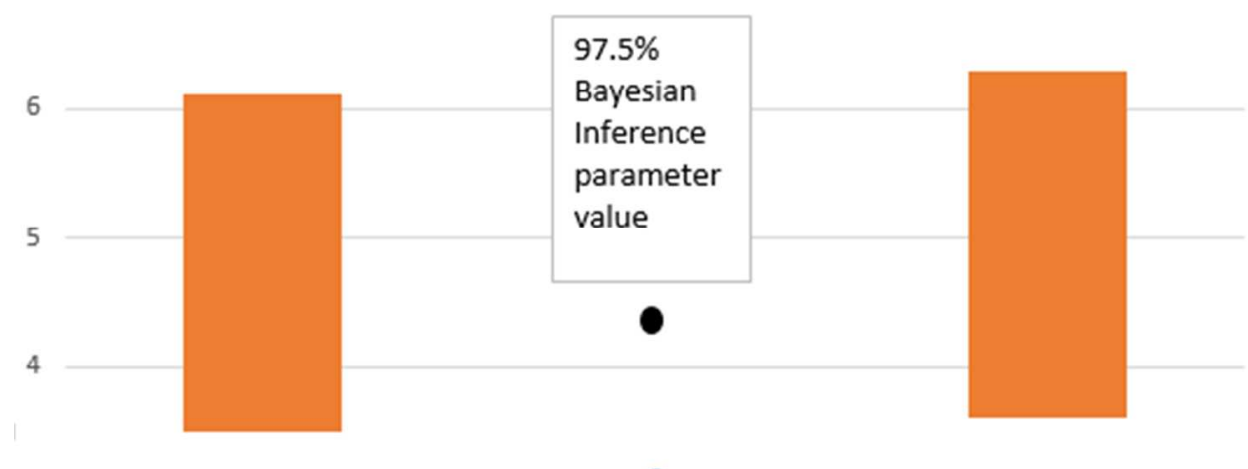

$3-95 \%$ $2 \quad \begin{aligned} & \text { confidence } \\ & \text { interval for }\end{aligned}$ Normal marginal,

1

$\mathrm{mpl}$ parameter estimation

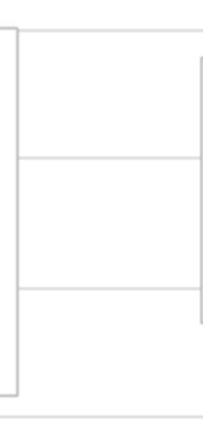

$95 \%$ confidence interval for GEV marginal, $\mathrm{mpl}$ parameter estimation

0

404 Figure 6 Comparison of parameter estimation based on maximized pseudolikelihood method 405 and Bayesian inference method The results from WinBUGS are shown in Fig. 7, 8, and 9, where the results of quantile, 407 kernel density, and autocorrelation values for theta are shown, respectively. 

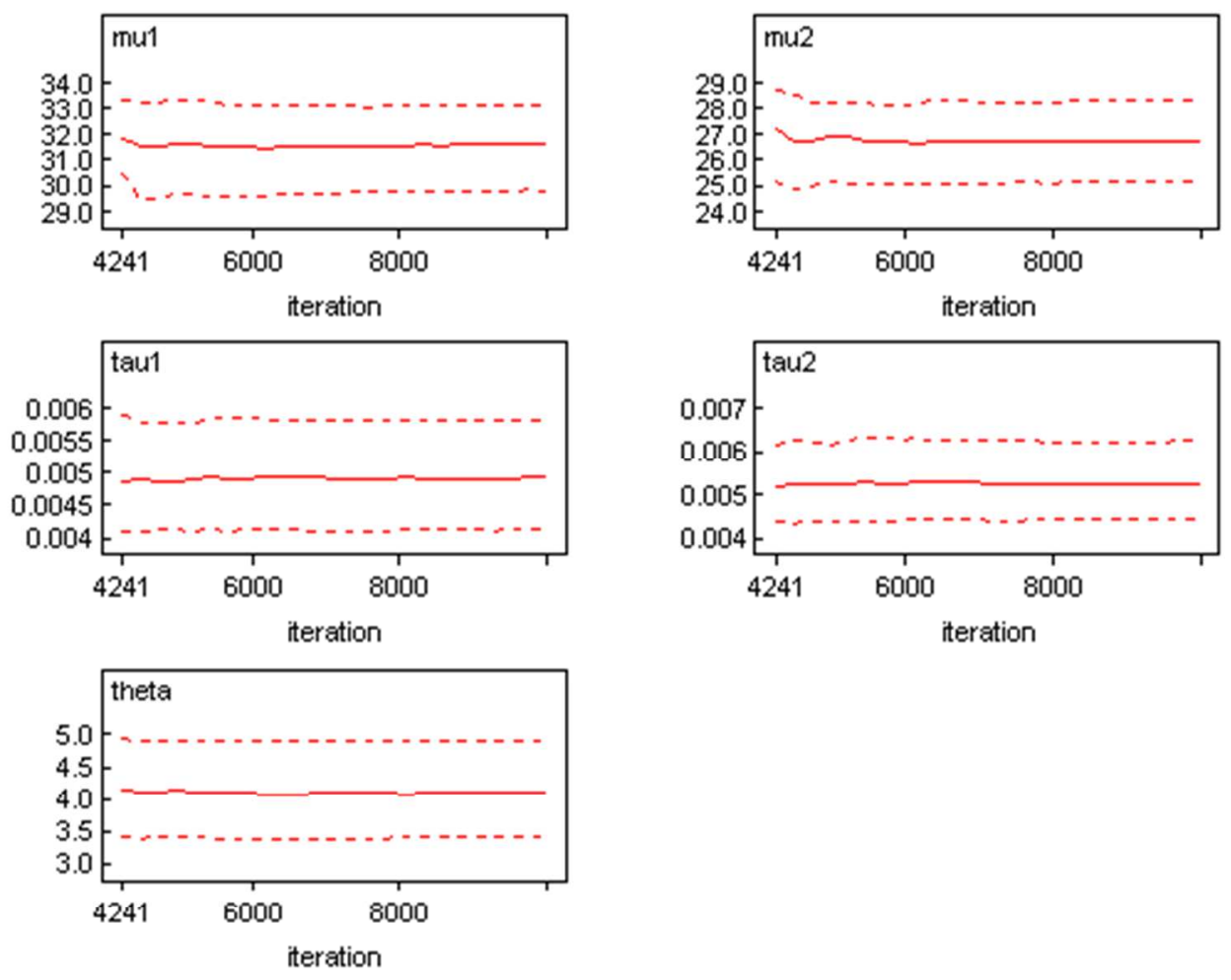

408

Figure 7. Quantile figure from WinBUGS analysis for Gumbel copula parameter estimation
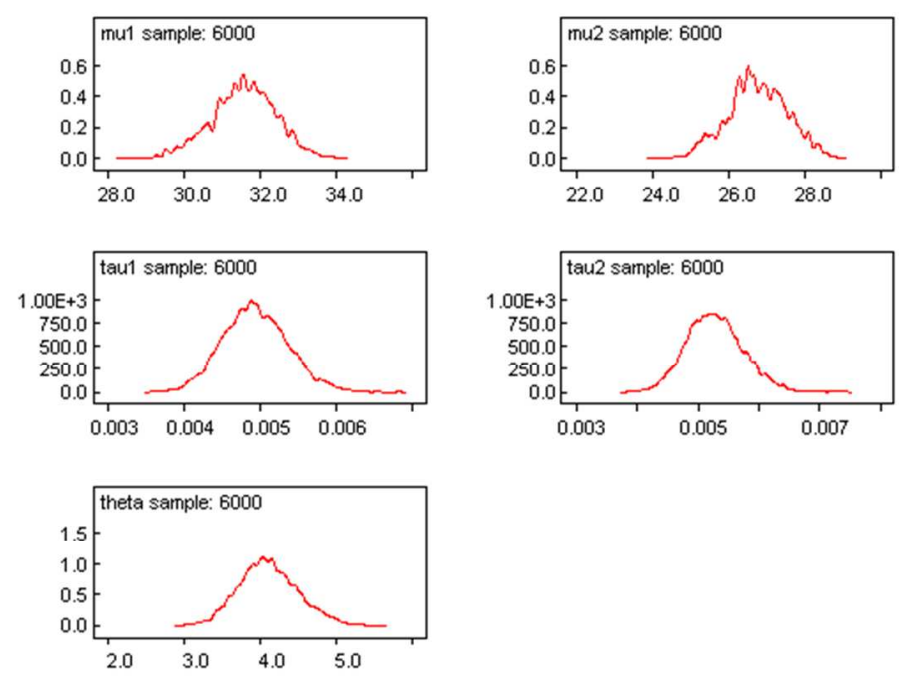

411

412 Figure 8. Density figure from WinBUGS analysis for Gumbel copula parameter estimation 


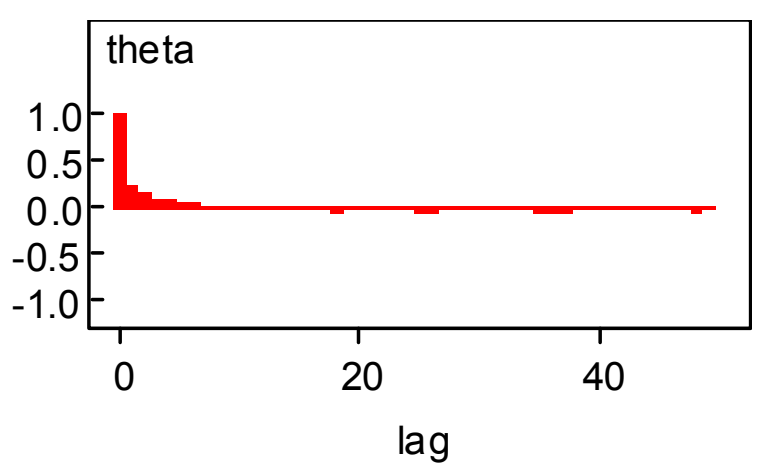

413

414 Figure 9. Convergence of simulation - autocorrelation plot

The trace plots for some of the parameters used in modeling are shown in Fig. 7. The

416 trace plots, which show the iterations versus generated values, are all within a parallel zone

417 without strong periodicities; thus, convergence can be said to have been reached. The

418 autocorrelation plot for the theta parameter is shown in Fig. 9. For the autocorrelation plot, there

419 is no obvious pattern with increasing lag, justifying the conclusion that convergence has been

420 reached. An important parameter used as a check on the simulation is the Monte Carlo (MC)

421 error. It measures the variability of the estimate due to the simulation. A low MC error is

422 required to calculate the parameter of interest with increased precision (Mills and Attoh-Okine

423 2014). From Table 6, for all the parameter calculations, the MC error value is significantly low.

\section{Frank Copula Parameter Estimation}

425 The bivariate Frank copula is expressed as

$$
C(u, v ; \theta)=\frac{1}{\theta} \ln \left(+\frac{\left(e^{-\theta u}-1\right)\left(e^{-\theta v}-1\right)}{e^{-\theta}-1}\right)
$$

427 Since the Frank copula is continuous, the bivariate copula density can be written as 
428

$$
c(u, v)=\frac{\partial^{2} C(u, v)}{\partial u \partial v}
$$

$$
=\frac{\theta\left(1-e^{-\theta}\right) e^{-\theta(u+v)}}{\left[1-e^{-\theta}-\left(1-e^{-\theta u}\right)\left(1-e^{-\theta v}\right)\right]^{2}}
$$

429

430

431

432

433

434

435

436

437

438

439

440

441 442 the $95 \%$ confidence level than the likelihood method. The summary for the result is shown in 443 Table 7. Mu1 and tau1 correspond to a value of mean and tau=1/std.dev ${ }^{2}$ of the pipe age

444 marginal.

For the Frank copula, a value of 50 for $\theta$ corresponds to a Spearman's rho of 0.99, which is almost equal to 1 . Therefore, a uniform distribution between 0 and 50 was used as the prior for theta. The normal distribution was chosen for the mean parameter, and the gamma distribution was chosen for the precision parameter tau.

corresponding to a Spearman's rho dependency of 0.936, to obtain the joint distribution. Fifty random samples were generated. This sample data was then used to obtain parameter $\theta$ by the maximized pseudolikelihood method for the Frank copula, which was 12.07 with a standard error of 2.086 . The $95 \%$ confidence level interval for the copula parameter yielded the lower limit of 7.98 and the upper limit of 16.16. Bayesian analysis was conducted to obtain parameter $\theta$. The result yielded a mean value of theta 11.25 with the 2.5 th and 97.5 th percentile values as 8.129 and 14.7, respectively. The Bayesian analysis result fit with a narrower variation within 
448 Table 7. Summary of results for Frank copula parameter

\begin{tabular}{llllllllll}
\hline 449 & node & mean & sd & MC error & $\mathbf{2 . 5 \%}$ & median & $\mathbf{9 7 . 5 \%}$ & start & sample \\
\cline { 2 - 9 } 450 & mu1 & 34.07 & 1.011 & 0.0289 & 32.08 & 34.08 & 36.07 & 4001 & 17000 \\
451 & mu2 & 27.66 & 0.9524 & 0.02786 & 25.77 & 27.64 & 29.56 & 4001 & 17000 \\
452 & tau1 & 0.007146 & $9.604 \mathrm{E}-4$ & $8.536 \mathrm{E}-6$ & 0.0054 & 0.007097 & 0.009143 & 4001 & 17000 \\
453 & tau2 & 0.008054 & 0.001074 & $9.752 \mathrm{E}-6$ & 0.00609 & 0.007995 & 0.01029 & 4001 & 17000 \\
454 & theta & $\mathbf{1 1 . 2 5}$ & $\mathbf{1 . 6 7 9}$ & $\mathbf{0 . 0 1 5 8 4}$ & $\mathbf{8 . 1 2 9}$ & $\mathbf{1 1 . 1 9}$ & $\mathbf{1 4 . 7}$ & 4001 & 17000 \\
\hline
\end{tabular}

From the above analysis, it is shown that Bayesian analysis gives a parameter close to the

457 original parameter of the chosen Frank copula. The values of the mean and standard deviation

458 are also close to original values of the marginals. This shows that Bayesian inference can be used

459 as an alternative method of parameter estimation for copula modeling. The copula parameter

460 estimation is a step of the modeling of non-gaussian pipe data shown in Figure 1, which in return

461 can generate large data set for water pipes.

462 Remarks

Infrastructure asset management is an approach that can help maintain utility at a desired

464 level of service at the lowest life-cycle cost. Asset management practices applied to underground

465 infrastructure can help utility companies understand the timing and cost associated with

466 rehabilitating, repairing, and replacing pipelines. Knowledge gained from these efforts also helps

467 to develop pipe material selection criteria. Pipe failure is a complex process involving the work

468 of many factors. It is important to have an understanding of the different factors that cause pipe 
469 leakage and to understand the common mechanisms of failure. Different pipe deterioration 470 models are applied to predict the condition of the water pipes.

471 In this research paper, copula modeling was applied to pipeline engineering. Copula modeling is

472 an emerging method of modeling and is useful in the case where the marginals belong to

473 different families of distributions. Copula modeling is also useful for generating large numbers

474 of data points when it is difficult to obtain data sets. This is the case for pipe condition

475 assessment, where underground pipe data information is expensive to obtain and where data sets

476 have random variables belonging to non-Gaussian family distributions. The large data sets

477 generated can then be used for evaluating the current pipe condition models and the

478 appropriateness of those models for determining the remaining life of a pipe or its condition.

479 In the pipe data set analysis presented here, it was shown that the general extreme value

480 distribution is a good fit for the marginals, and data simulated with these marginals provide more

481 accurate values for regression analysis for future predictions. The parameter estimation of a

482 copula can be achieved through different methods, such as maximized likelihood, inversion of

483 Spearman's rho, inversion of Kendall's tau, and maximized pseudolikelihood, among which the

484 maximized pseudolikelihood method is based on ranked data. These parameter estimation

485 methods are newly developed methods. Bayesian inference is an alternative method that can be

486 used to obtain the parameters of the copulas. The results indicated that Bayesian inference can

487 provide a good estimate of copula parameters. Two types of copula, the Frank and Gumbel

488 copulas, were tested with a normal marginal, and both gave good estimations. In comparison to

489 the pseudolikelihood method of parameter estimation, the Bayesian result provided a narrower

490 range for the parameter value. 
492 maximize directly. In such cases, Bayesian inference using MCMC is a better alternative. For

493 further research, the analysis can be extended to include methods applying Bayesian inference to

494 choose a model of different copulas to fit the pipe condition data based on the Akaike

495 information criterion (AIC).

496

497

498

499

500

501

502

503

504

505

506

507 


\section{References}

Accioly, R., and Chiyoshi, F.Y. 2004. "Modeling dependence with copulas, a useful tool for field development decision process." Journal of Petroleum Science Engineering, 44, 83-91.

Atique, F., and Attoh-Okine, N. 2016. "Using copula method for pipe data analysis." Construction and Building Materials, 106, 140-148.

Attoh-Okine, N. 2013. "Pair-copulas in infrastructure multivariate dependence modeling." Construction and Building Materials, 49, 903-911.

Bacigal, T. 2006. "Fitting Archimedean copulas to bivariate geodetic observations. " $5^{\text {th }}$ International Conference APLIMAT, (add sponsor/organization name), Bratislava, Slovakia, 8189.

Berg, D. 2008. Using copulas: An introduction to practitioners, Norwegian Actuarial Studies In Non-life insurance (ASTIN) Society, Oslo, Norway.

Embrechts, P., Lindkog, F., and McNeil, A. 2001. Risk Lab, ETH Zurich, Zurich, Switzerland.

Frees, E.W., and Valdez, E.A. 1998. "Understanding relationships using copulas.” North American Actuarial J., 2(1), 1-25.

Folkman, S. 2012. Water main break rates in the USA and Canada: A comprehensive study, Utah State University Buried Structures Laboratory, Logan, Utah.

Genest, C., and Favre, A. 2007. "Everything you always wanted to know about copula modeling but were afraid to ask." Journal of Hydrologic Engineering, 12, 347-368.

Genest, C., Ghoudi, K., and Rivest, L.P. (1995). "A semiparametric estimation procedure of dependence parameters in multivariate families of distribution." Biometrika, 82(3), 545-552.

Kleiner, Y., and Rajani, B. 2001a. "Comprehensive review of structural deterioration of water mains: Statistical methods.” Urban Water, 3, 131-150.

Kleiner, Y., and Rajani, B. 2001b. "Comprehensive review of structural deterioration of water mains: Physically based model.” Urban Water, 3, 151-164.

Kelly, D.L. 2007. "Using copulas to model dependence in simulation risk assessment." 2007 ASME International Mechanical Engineering Congress and Exposition, ASME, Seattle, Washington, (add page numbers).

Mills, L. O., and Attoh-Okine, N. 2014. "Analysis of ground penetrating radar data using hierarchical Markov Chain Monte Carlo simulation." Canadian Journal of Civil Engineering, 41(1), 9-16. 
553

554

555

556

557

558

559

560

561

562

563

564

565

566

567

568

569

570

571

572

573

574

575

576

577

578

579

580

581

582
Naimy, V.Y., (2012) Gaussian Copula vs Loans Loss Assessment: A Simplified and Easy-ToUse Model. Journal of Business \& Financial Affairs , 1:105

Nelsen, R.B. 1999. An introduction to copulas, Springer, New York.

Opila, M. C., and Attoh-Okine, N. 2011. "Novel approach in pipe condition scoring." Journal of Pipeline Systems Engineering and Practice, 2(3), 82-90.

Romeo, J.S., Tanaka T.I., and Pedroso-de-Lima, A.C. 2006. "Bivariate survival modelling: A Bayesian approach based on copulas." Lifetime Data Analysis, 12, 205-222.

Scheidegger, A., Hug, T., Rieckermann, J., and Maurer, M. 2011. "Network condition simulator for benchmarking sewer deterioration models." Water Research, 45(16), 4983-4994.

Silva, R.D.S., and Lopes, H.F. 2008. "Copula marginal distributions and model selection: A Bayesian note." Statistical Computation, 18, 313-320.

Smith, M.S. 2011. "Bayesian approaches to copula modeling." In Bayesian theory and applications, Paul Damien, Petros Dellaportas, Nicholas G. Polson, and David A. Stephens, eds. Oxford University Press, Oxford, United Kingdom, 336.

Srinivas, S., Menon, D., and Prasad, A.M. 2006. "Multivariate simulation and multimodal modelling of vehicle axle weights with copulas." Journal of Transportation Engineering, 132(12), 945-955.

Yan, J. 2006. "Multivariate modeling with copulas and engineering applications." In Springer handbook of engineering statistics, Hoang Pham, ed. Springer, London, 973-990. 